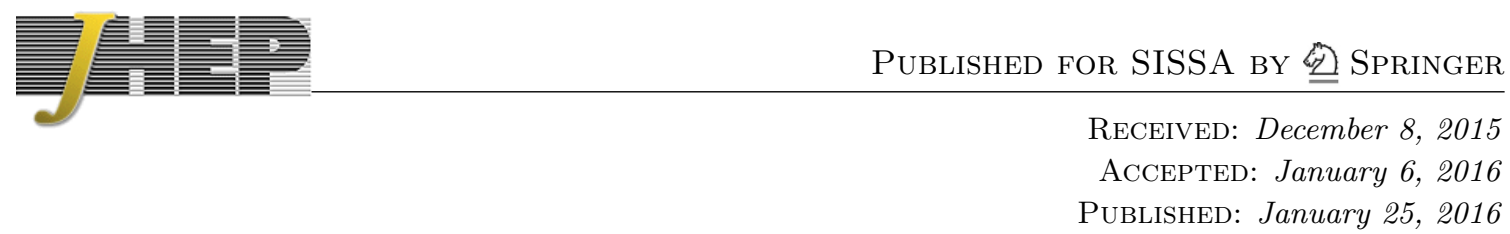

\title{
Lax pairs for deformed Minkowski spacetimes
}

\author{
Hideki Kyono, Jun-ichi Sakamoto and Kentaroh Yoshida \\ Department of Physics, Kyoto University, \\ Kitashirakawa Oiwake-cho, Kyoto 606-8502, Japan \\ E-mail: h_kyono@gauge.scphys.kyoto-u.ac.jp, \\ sakajun@gauge.scphys.kyoto-u.ac.jp, \\ kyoshida@gauge.scphys.kyoto-u.ac.jp
}

ABSTRACT: We proceed to study Yang-Baxter deformations of 4D Minkowski spacetime based on a conformal embedding. We first revisit a Melvin background and argue a Lax pair by adopting a simple replacement law invented in 1509.00173. This argument enables us to deduce a general expression of Lax pair. Then the anticipated Lax pair is shown to work for arbitrary classical $r$-matrices with Poincaré generators. As other examples, we present Lax pairs for pp-wave backgrounds, the Hashimoto-Sethi background, the SpradlinTakayanagi-Volovich background.

KeYwords: Integrable Field Theories, Sigma Models

ArXiv EPrint: 1512.00208 


\section{Contents}

1 Introduction 1

2 Yang-Baxter deformations of Minkowski spacetime 3

3 A Melvin background revisited $\quad 5$

3.1 Lax pair for 4D Minkowski spacetime 5

$\begin{array}{ll}3.2 & \text { Lax pair for a Melvin background }\end{array}$

$\begin{array}{lll}3.3 & \text { Twisted boundary condition } & 7\end{array}$

4 General arguments $\quad 8$

4.1 Proof of the anticipated Lax pair 8

$\begin{array}{ll}4.2 \text { Twisted boundary condition } & 10\end{array}$

$\begin{array}{ll}4.3 & \text { The case of mCYBE } \\ \end{array}$

5 Other examples $\quad 13$

$\begin{array}{lll}5.1 & \text { pp-wave backgrounds } & 13\end{array}$

$\begin{array}{ll}5.2 \text { Hashimoto-Sethi background } & 14\end{array}$

$\begin{array}{lll}5.3 & \text { Spradlin-Takayanagi-Volovich background } & 16\end{array}$

6 Conclusion and discussion $\quad 18$

$\begin{array}{lr}\text { A Notation and convention } & 18\end{array}$

$\begin{array}{ll}\text { B A conformal embedding of 4D Minkowski spacetime } & 19\end{array}$

\section{Introduction}

The Yang-Baxter sigma-model description is known as a systematic way to study integrable deformations of $2 \mathrm{D}$ integrable non-linear sigma models [1-3]. It was originally proposed for principal chiral models on the basis of the modified classical Yang-Baxter equation (mCYBE) . It was generalized to symmetric cosets by Delduc, Magro and Vicedo [4] and then to the homogeneous classical Yang-Baxter equation (CYBE) [5].

Yang-Baxter deformations can be applied to integrable deformations of the $\operatorname{AdS}_{5} \times \mathrm{S}^{5}$ superstring. The first example is a $q$-deformation of the $\mathrm{AdS}_{5} \times \mathrm{S}^{5}$ superstring $[6,7]$ based on the mCYBE. This deformations is often called the $\eta$-deformation. The metric and NS-NS two-form are obtained in [8]. The remaining fields are determined recently by performing a super coset construction at the level of quadratic fermions [9]. As a result, the obtained fields do not satisfy the on-shell condition of type IIB supergravity. (For another approach to an exact solution including T-duals of the metric, see [10]). However, 
in the very recent paper [11], it is shown that the scale invariance of the world-sheet theory is still preserved and it has been conjectured the background satisfies rather the modified type IIB supergravity equations of motion in which the RR-field strengths are of the second order in derivatives.

Another class of Yang-Baxter deformations of the $\operatorname{AdS}_{5} \times \mathrm{S}^{5}$ superstring is based on the homogeneous CYBE [12]. The deformations include TsT transformations of $\operatorname{AdS}_{5} \times \mathrm{S}^{5}$ [13$15]$ such as $\gamma$-deformations of $S^{5}[16,17]$, gravity duals of noncommutative gauge theories [18, 19], and Schrödinger geometries [20-22]. These backgrounds can also be realized as the undeformed $\mathrm{AdS}_{5} \times \mathrm{S}^{5}$ with twisted boundary conditions [17, 23-30]. Other deformations are associated with classical $r$-matrices composed of non-commuting generators and lead to deformed backgrounds obtained through a chain of dualities including S-dualities [31, 32]. Further remarkably, these deformations may work for non-integrable backgrounds, such as a Sasaki-Einstein manifold $T^{1,1}[33,34]$. TsT transformations of $T^{1,1}[16,35]$ are reproduced as Yang-Baxter deformations [36, 37]. Thus the connection between gravity solutions and classical $r$-matrices may deserve to be called the gravity/CYBE correspondence (For a short summary see [38]).

Furthermore, we have considered Yang-Baxter deformations of 4D Minkowski spacetime as a generalization, in order to examine applicability of the correspondence $[39,40] .{ }^{1}$ Firstly, the work [39] showed that the Yang-Baxter deformations can reproduce twisted backgrounds such as Melvin backgrounds [42-45], pp-wave backgrounds [46] and timedependent exactly-solvable backgrounds including the Hashimoto-Sethi background [47] and the Spradlin-Takayanagi-Volovich background [48], like in the case of $\operatorname{AdS}_{5} \times \mathrm{S}^{5}$. Then the analysis has been generalized by adopting classical $r$-matrices which describe $\kappa$-deformations of Poincaré algebra $[49,50]$. The associated deformed backgrounds include T-duals of $(\mathrm{A}) \mathrm{dS}_{4}$ and a time dependent pp-wave background. Interestingly, these backgrounds can also be reproduced from different classical $r$-matrices including the dilatation [39]. In addition, the Lax pair has been constructed for the general $\kappa$-deformations [40].

The construction of the Lax pair in [40] utilized the expression of Lax pair for arbitrary classical $r$-matrices which are composed of Poincaré generators and satisfy the CYBE, while the derivation of this part has not been explained. Our main purpose here is to provide the derivation as a follow-up of the previous work [40]. We first revisit a Melvin background and argue the associated Lax pair by following a simple replacement law invented in [30]. This argument enables us to deduce a general expression of Lax pair. Then we show that the anticipated Lax pair works for arbitrary classical $r$-matrices with Poincaré generators. As other examples, we present Lax pairs for pp-wave backgrounds, the Hashimoto-Sethi background, the Spradlin-Takayanagi-Volovich background.

This parer is organized as follows. In section 2, we give a brief review of Yang-Baxter deformations of 4D Minkowski spacetime. In section 3, a Melvin background is revisited and the associated Lax pair is constructed by following a simple replacement rule invented in [30]. A twisted boundary condition is also discussed. In section 4, we show that the anticipated Lax pair works for arbitrary classical $r$-matrices which are composed of Poincaré

\footnotetext{
${ }^{1}$ For an approach based on a scaling limit of $q$-deformed $\mathrm{AdS}_{5} \times \mathrm{S}^{5}$, see [41].
} 
generators. In section 5, we present Lax pairs and argue twisted boundary conditions for the following examples: 1) pp-wave backgrounds, 2) the Hashimoto-Sethi background, 3) the Spradlin-Takayanagi-Volovich background. Section 6 is devoted to conclusion and discussion. In appendix A, our notation and convention are summarized. Appendix B gives a short explanation of a conformal embedding of 4D Minkowski spacetime.

\section{Yang-Baxter deformations of Minkowski spacetime}

We shall present a short review of Yang-Baxter deformations of 4D Minkowski spacetime by following the previous works [39, 40]. For our notation and convention, see appendix A.

A significant point is that the problem of the degenerate Killing form can be avoided by adopting a conformal embedding as briefly described in appendix B. In the following, the string tension $T=\frac{1}{2 \pi \alpha^{\prime}}$ is set to 1 , and the conformal gauge is taken so as to drop the dilaton coupling to the world-sheet scalar curvature.

The Yang-Baxter deformed action is given by [39]

$$
S=-\frac{1}{2} \int_{-\infty}^{\infty} d \tau \int_{0}^{2 \pi} d \sigma\left(\gamma^{\alpha \beta}-\epsilon^{\alpha \beta}\right) \operatorname{Tr}\left[A_{\alpha} P \circ \frac{1}{1-2 \eta R_{g} \circ P} A_{\beta}\right] .
$$

The left-invariant one-form $A_{\alpha} \equiv g^{-1} \partial_{\alpha} g$ is defined with a group element $g$ given in (B.1) . The projection $P$ is defined as

$$
P(x) \equiv \frac{1}{4}\left[-\gamma_{0} \operatorname{Tr}\left(\gamma_{0} x\right)+\sum_{i=1}^{3} \gamma_{i} \operatorname{Tr}\left(\gamma_{i} x\right)\right]
$$

and is closely related to the coset structure of $\mathrm{AdS}_{5}$. The deformation is measured by $\eta$. The undeformed action is reproduced when $\eta=0$. The world-sheet metric is given by $\gamma_{\alpha \beta}=\operatorname{diag}(-1,1)$. The anti-symmetric tensor $\epsilon^{\alpha \beta}$ is normalized as $\epsilon^{\tau \sigma}=1$.

A key ingredient is a linear $R$-operator contained in $R_{g}$ defined as

$$
R_{g} \equiv g^{-1} R\left(g X g^{-1}\right) g \text {. }
$$

The linear operator $R: \mathfrak{s o}(2,4) \rightarrow \mathfrak{s o}(2,4)$ is a solution of the mCYBE $[51,52]$,

$$
[R(x), R(y)]-R([R(x), y]+[x, R(y)])=\omega[x, y], \quad x, y \in \mathfrak{s o}(2,4),
$$

where $\omega$ is a constant parameter. The $R$-operator corresponds to a skew-symmetric classical $r$-matrix in the tensorial notation through the following formula:

$$
R(X)=\operatorname{Tr}_{2}[r(1 \otimes X)]=\sum_{i}\left(a_{i} \operatorname{Tr}\left(b_{i} X\right)-b_{i} \operatorname{Tr}\left(a_{i} X\right)\right)
$$

Here the classical $r$-matrix is represented by

$$
r=\sum_{i} a_{i} \wedge b_{i} \equiv \sum_{i}\left(a_{i} \otimes b_{i}-b_{i} \otimes a_{i}\right) .
$$


The generators $a_{i}, b_{i}$ are elements of $\mathfrak{s o}(2,4)$. This means that Yang-Baxter deformations are investigated based on the extended algebra $\mathfrak{s o}(2,4)$, rather than the Poincaré algebra.

In particular, when $\omega=0$, the equation (2.4) is called the homogenous CYBE. When (skew-symmetric) classical $r$-matrices satisfy the standard CYBE,

$$
\left[r_{12}, r_{13}\right]+\left[r_{12}, r_{23}\right]+\left[r_{13}, r_{23}\right]=0
$$

they lead to $R$-operators which satisfy the homogenous CYBE. In the following, we are mainly concerned with the homogeneous CYBE case.

To construct Lax pairs, it is useful to work with the following deformed current,

$$
J_{ \pm}=\frac{1}{1 \mp 2 \eta R_{g} \circ P}\left(A_{ \pm}\right) .
$$

It is also helpful to introduce the world-sheet light-cone components as

$$
A_{ \pm} \equiv A_{\tau} \pm A_{\sigma}
$$

Then the Lagrangian in (2.1) can be rewritten into a simpler form:

$$
L=\frac{1}{2} \operatorname{Tr}\left[A_{-} P\left(J_{+}\right)\right]
$$

One can read off the deformed metric and NS-NS two-form from the symmetric and skew symmetric parts with respect to the world-sheet coordinates in the deformed Lagrangian, respectively.

It should be remarked that, although it is sufficient to compute an explicit form of the projected deformed current $P(J)$ to study the deformed metric, the explicit form of the deformed current $J$ itself is necessary to construct Lax pairs.

A schematic classification of classical $r$-matrices. It would be helpful to list up possible classical $r$-matrices concerned with our analysis. Schematically, those are classified into the following three classes:

(a) $\quad r=$ Poincaré $\otimes$ Poincaré

1. abelian e.g., $\quad r \sim p_{1} \wedge p_{2}, \quad$ 2. non-abelian e.g., $\quad r \sim \sum_{i=1}^{3} p_{i} \wedge n_{0 i}$,

(b) $\quad r=$ Poincaré $\otimes$ non-Poincaré

1. abelian e.g., $\quad r \sim n_{12} \wedge \hat{d}, \quad$ 2. non-abelian e.g., $\quad r \sim p_{0} \wedge \hat{d}$,

(c) $\quad r=$ non-Poincaré $\otimes$ non-Poincaré
1. abelian e.g., $\quad r \sim k_{1} \wedge k_{2}$,
2. non-abelian e.g., $\quad r \sim k_{0} \wedge \hat{d}$.

Note that, given a classical $r$-matrix $r=a \otimes b$, the word "abelian" means that a and $\mathrm{b}$ commute each other. ${ }^{2}$ The generators of $4 \mathrm{D}$ conformal algebra $p_{\mu}, n_{\mu \nu}, k_{\mu}$ and $\hat{d}$ are associated with translations, Lorentz rotations, special conformal transformations and dilatation, respectively (For our notation and convention, see appendix A).

\footnotetext{
${ }^{2}$ In the case that summations are included, one needs to be careful for the definition in detail. But we will not try to dwell on it here.
} 
The complete classification of classical $r$-matrices has not been done and the associated Yang-Baxter deformations have also not been investigated completely. It is nice to look for the relation between classical $r$-matrices and deformed geometries furthermore. For classical $r$-matrices in the class (a), it would be helpful to consult an intensive classification in $[53-55]$.

So far, classical $r$-matrices in the classes (a)-1 and (b)-2 have been studied in [39, 40]. The class (a)-1 is intimately related to (generalized) Melvin twists [39]. Some examples of the class (b)-2 have been studied as well. In particular, T-duals of $\mathrm{dS}_{4}$ and $\mathrm{AdS}_{4}$ are realized with $r \sim p_{0} \wedge \hat{d}$ and $r \sim p_{1} \wedge \hat{d}$, respectively. Interestingly, special classical $r$-matrices in the class (a)-2, which are also used in the context of $\kappa$-deformations of Poincaré algebra, lead to the identical T-duals of $\mathrm{dS}_{4}$ and $\mathrm{AdS}_{4}$ [40].

Although we have succeeded in constructing a Lax pair for the general $\kappa$-deformations, the construction was based on the expression of Lax pair for the class (a) with the homogeneous CYBE and we have not derived it explicitly.

In the following, we will present a method to construct Lax pairs for the classical $r$ matrices in the class (a) that satisfy the homogeneous CYBE. A simple replacement law based on TsT transformations is utilized to deduce a general form of Lax pair for arbitrary classical $r$-matrices in the class (a)-1. Finally, the anticipated Lax pair is shown to work for all classical $r$-matrices in the class (a) with the homogeneous CYBE. It is straightforward to generalize the general form to the mCYBE case, by following the previous work [40].

\section{A Melvin background revisited}

Let us revisit a Melvin background here. We derive a Lax pair by applying a simple replacement rule to the one for $4 \mathrm{D}$ Minkowski spacetime. Then the result enables us to deduce a general form of Lax pair for an arbitrary classical $r$-matrix of the class (a)- 1 . We also argue a general form of twisted boundary condition with which the undeformed background is equivalent to the deformed background.

\subsection{Lax pair for 4D Minkowski spacetime}

We first construct the Lax pairs for sigma models on 4D Minkowski spacetime. The starting classical action can be expressed as

$$
S=\frac{1}{2} \int_{-\infty}^{\infty} d \tau \int_{0}^{2 \pi} d \sigma \operatorname{Tr}\left[A_{-} P\left(A_{+}\right)\right]
$$

Then the associated Lax pair is given by

$$
\mathcal{L}_{ \pm}^{\mathrm{Min}}=P_{0}\left(A_{ \pm}\right)+\lambda^{ \pm 1} P_{2}\left(A_{ \pm}\right)
$$

with a spectral parameter $\lambda \in \mathbb{C}$. Here $P_{0}$ and $P_{2} \equiv P+P^{\prime}$ are the projection operators, where $P$ is already introduced in (2.2), and $P_{0}$ and $P^{\prime}$ are defined as, respectively,

$$
P_{0}(x) \equiv \frac{1}{2} \sum_{\mu, \nu=0}^{3} n_{\mu \nu} \frac{\operatorname{Tr}\left(n_{\mu \nu} x\right)}{\operatorname{Tr}\left(n_{\mu \nu} n_{\mu \nu}\right)}, \quad P^{\prime}(x) \equiv \sum_{\mu=0}^{3} n_{\mu 5} \frac{\operatorname{Tr}\left(n_{\mu 5} x\right)}{\operatorname{Tr}\left(n_{\mu 5} n_{\mu 5}\right)} .
$$


For the standard coset representative (B.1), it is easy to check that the equations of motion are equivalent to the flatness condition of the Lax pair (3.2). Note that $P_{0}$ and $P_{2}$ are regarded as the projections to the $\mathbb{Z}_{2}$-graded components of $4 \mathrm{D}$ Poincaré algebra iso $(1,3)$,

$$
\begin{aligned}
& P_{0}: \quad \mathfrak{i s o}(1,3) \quad \longrightarrow \quad \mathfrak{i s o}^{(0)}(1,3)=\mathfrak{s o}(1,3), \\
& P_{2}: \quad \mathfrak{i s o}(1,3) \quad \longrightarrow \quad \mathfrak{i s o}^{(2)}(1,3)=\mathfrak{i s o}(1,3) / \mathfrak{s o}(1,3) .
\end{aligned}
$$

\subsection{Lax pair for a Melvin background}

Next, we will derive a Lax pair for a Melvin background [42-45] associated with an abelian classical $r$-matrix

$$
r=\frac{1}{2} p_{3} \wedge n_{12}
$$

This $r$-matrix leads to the metric,

$$
\begin{array}{rlrl}
d s^{2} & =-\left(d x^{0}\right)^{2}+d r^{2}+G_{\mathrm{M}}\left[r^{2} d \theta^{2}+\left(d x^{3}\right)^{2}\right], \\
B & =\eta r^{2} G_{\mathrm{M}} d \theta \wedge d x^{3}, & G_{\mathrm{M}}^{-1} \equiv 1+\eta^{2} r^{2},
\end{array}
$$

with the standard coset representative (B.1) and the polar coordinates

$$
x^{1}=r \cos \theta, \quad x^{2}=r \sin \theta .
$$

This background describes an example of Melvin backgrounds.

Now that the left-invariant one-form $A$ can be expanded as

$$
A_{ \pm}=\partial_{ \pm} x^{0} p_{0}+\left[\cos \theta \partial_{ \pm} r-r \sin \theta \partial_{ \pm} \theta\right] p_{1}+\left[\sin \theta \partial_{ \pm} r+r \cos \theta \partial_{ \pm} \theta\right] p_{2}+\partial_{ \pm} x^{3} p_{3}
$$

the deformed current $J$ is evaluated as

$$
\begin{aligned}
J_{ \pm}= & \partial_{ \pm} x^{0} p_{0}+G_{\mathrm{M}}\left(\partial_{ \pm} x^{3} \mp \eta r^{2} \partial_{ \pm} \theta\right)\left(p_{3}-\eta n_{12}\right) \\
& +\left[\cos \theta \partial_{ \pm} r-G_{\mathrm{M}} r \sin \theta\left(\partial_{ \pm} \theta \pm \eta \partial_{ \pm} x^{3}\right)\right] p_{1} \\
& +\left[\sin \theta \partial_{ \pm} r+G_{\mathrm{M}} r \cos \theta\left(\partial_{ \pm} \theta \pm \eta \partial_{ \pm} x^{3}\right)\right] p_{2}
\end{aligned}
$$

Comparing $J$ with $A$, one can find the following replacement rule:

$$
\begin{aligned}
& \partial_{ \pm} \theta \quad \longrightarrow \quad G_{\mathrm{M}}\left(\partial_{ \pm} \theta \pm \eta \partial_{ \pm} x^{3}\right), \\
& \partial_{ \pm} x^{3} \quad \longrightarrow \quad G_{\mathrm{M}}\left(\partial_{ \pm} x^{3} \mp \eta r^{2} \partial_{ \pm} \theta\right) \text {. }
\end{aligned}
$$

Note that this rule makes sense at the off-shell level.

One can derive a Lax pair for the Melvin background (3.6) by applying the replacement rule (3.10) to the undeformed one. First of all, it is necessary to prepare the undeformed Lax pair that has a manifest $\mathrm{U}(1)$ isometry along the $\theta$-direction. It is easy to remove the $\theta$-dependence of the undeformed Lax by performing a gauge transformation,

$$
\begin{aligned}
\mathcal{L}_{ \pm}^{\mathrm{Min}} \rightarrow \mathcal{L}_{ \pm}^{\mathrm{Min}, h} & =h^{-1} \mathcal{L}_{ \pm}^{\mathrm{Min}} h+h^{-1} \partial_{ \pm} h \\
& =-\partial_{ \pm} \theta n_{12}+\lambda^{ \pm 1}\left(\partial_{ \pm} r p_{1}+r \partial_{ \pm} \theta p_{2}+\partial_{ \pm} x^{3} p_{3}+\partial_{ \pm} x^{0} p_{0}\right)
\end{aligned}
$$


with $h=\exp \left(-\theta n_{12}\right)$. The resulting Lax pair depends only on the derivative of $\theta$ and hence has a $\mathrm{U}(1)$ symmetry along the $\theta$-direction. Thus, by applying the replacement rule (3.10) to (3.11), the Lax pair for the Melvin background is given by

$$
\begin{aligned}
\mathcal{L}_{ \pm}^{\text {Melvin }}= & -G_{\mathrm{M}}\left(\partial_{ \pm} \theta \pm \eta \partial_{ \pm} x^{3}\right) n_{12} \\
& +\lambda^{ \pm 1}\left[\partial_{ \pm} x^{0} p_{0}+\partial_{ \pm} r p_{1}+G_{\mathrm{M}} r\left(\partial_{ \pm} \theta \pm \eta \partial_{ \pm} x^{3}\right) p_{2}+G_{\mathrm{M}}\left(\partial_{ \pm} x^{3} \mp \eta r^{2} \partial_{ \pm} \theta\right) p_{3}\right]
\end{aligned}
$$

One can readily check that the zero-curvature condition of this Lax pair is equivalent to the equations of motion for the Melvin background.

Now we can deduce a general form of Lax pair for arbitrary classical $r$-matrices in the class (a)-1. First, with the deformed current $J$ in (3.9), the Lax pair (3.12) can be rewritten into the following form:

$$
\mathcal{L}_{ \pm}^{\text {Melvin }}=h^{-1} \partial_{ \pm} h+h^{-1} P_{0}\left(J_{ \pm}\right) h+\lambda^{ \pm 1} h^{-1} P_{2}\left(J_{ \pm}\right) h .
$$

The flatness condition of the Lax pair should be preserved under arbitrary gauge transformations. Thus the resulting Lax pair is given by

$$
\mathcal{L}_{ \pm}^{\text {Melvin }}=P_{0}\left(J_{ \pm}\right)+\lambda^{ \pm 1} P_{2}\left(J_{ \pm}\right) .
$$

This abstract expression is really significant because one can anticipate that the form (3.14) would hold for arbitrary classical $r$-matrices in the class (a)-1 satisfying the homogeneous CYBE. In fact, this anticipation is true as proven in the next section. More interestingly, the Lax pair (3.14) works for the class (a)-2 as well. Thus, in total, the Lax pair (3.14) holds for arbitrary classical $r$-matrices in the class (a) satisfying the homogeneous CYBE.

\subsection{Twisted boundary condition}

The Melvin background (3.6) can be realized as a Melvin twist of 4D Minkowski spacetime [42]. We will reconsider here the Melvin background (3.6) from the point of view of a twisted boundary condition for 4D Minkowski spacetime.

For this purpose, it is useful to take the following coset representative:

$$
\begin{aligned}
g(\tau, \sigma) & =\exp \left[x^{3} p_{3}-\theta n_{12}\right] \exp \left[x^{0} p_{0}+r p_{1}\right] \\
& =\exp \left[x^{0} p_{0}+r \cos \theta p_{1}+r \sin \theta p_{2}+x^{3} p_{3}\right] \exp \left[-\theta n_{12}\right] .
\end{aligned}
$$

This representative (3.15) is equivalent to the standard one (B.1) under the gauge transformation (3.11) .

Then, with the replacement rule (3.10), we will show that the deformed sigma model can be mapped to the undeformed one with a twisted boundary condition. In the first place, one needs to obtain U(1) currents in the undeformed and deformed backgrounds, respectively,

$$
\begin{aligned}
\tilde{\mathcal{J}}_{\theta}^{\alpha} & =-\gamma^{\alpha \beta} r^{2} \partial_{\beta} \tilde{\theta}, \\
\tilde{P}_{3}^{\alpha} & =-\gamma^{\alpha \beta} \partial_{\beta} \tilde{x}^{3} \\
\mathcal{J}_{\theta}^{\alpha} & =-\gamma^{\alpha \beta} G_{M} r^{2}\left[\partial_{\beta} \theta-\eta \gamma_{\beta \gamma} \epsilon^{\gamma \rho} \partial_{\rho} x^{3}\right], \\
P_{3}^{\alpha} & =-\gamma^{\alpha \beta} G_{M}\left[\partial_{\beta} x^{3}+\eta \gamma_{\beta \gamma} \epsilon^{\gamma \rho} r^{2} \partial_{\rho} \theta\right] .
\end{aligned}
$$


Here $\tilde{x}^{3}$ and $\tilde{\theta}$ represent coordinates on the undeformed background. With the rule (3.10), one can show that the $\mathrm{U}(1)$ currents in $4 \mathrm{D}$ Minkowski spacetime are equal to those in the Melvin background, i.e.,

$$
\tilde{\mathcal{J}}_{\theta}^{\alpha}=\mathcal{J}_{\theta}^{\alpha}, \quad \tilde{P}_{3}^{\alpha}=P_{3}^{\alpha} .
$$

The temporal components of the relations in (3.17) imply that $P_{3}^{\tau}$ and $\mathcal{J}_{\theta}^{\tau}$ are equivalent to $\tilde{\mathcal{J}}_{\theta}^{\tau}$ and $\tilde{P}_{3}^{\tau}$. Then, by integrating the spacial components of the relations in (3.17), twisted boundary conditions for the undeformed coordinates $\tilde{\theta}$ and $\tilde{x}^{3}$ are obtained as

$$
\begin{aligned}
\tilde{\theta}(\tau, 2 \pi) & =\tilde{\theta}(\tau, 0)+\eta P_{3}+2 \pi w, \\
\tilde{x}^{3}(\tau, 2 \pi) & =\tilde{x}^{3}(\tau, 0)-\eta \mathcal{J}_{\theta} .
\end{aligned}
$$

Here $w \in \mathbb{Z}$ is a winding number for the angular coordinate $\theta$. The conserved charges $P_{3}$ and $\mathcal{J}_{\theta}$ are defined as

$$
P_{3} \equiv \int_{0}^{2 \pi} d \sigma P_{3}^{\tau}, \quad \mathcal{J}_{\theta} \equiv \int_{0}^{2 \pi} d \sigma \mathcal{J}_{\theta}^{\tau}
$$

Note here that there is a relation between a gauge transformed current $J$

$$
J_{\alpha}^{g}=g J_{\alpha} g^{-1}+g \partial_{\alpha} g^{-1}
$$

and the $\mathrm{U}(1)$ currents

$$
\begin{aligned}
J_{\sigma}^{g} & =-\eta r^{2} G_{\mathrm{M}}\left(\dot{\theta}+\eta x^{\prime 3}\right) p_{3}-\eta G_{\mathrm{M}}\left(\dot{x}^{3}-\eta r^{2} \theta^{\prime}\right) n_{12} \\
& =-\eta \mathcal{J}_{\theta}^{\tau} p_{3}-\eta P_{3}^{\tau} n_{12} .
\end{aligned}
$$

Thus the twisted boundary conditions (3.18) can be recast into the following form:

$$
\tilde{g}(\tau, 2 \pi)=\mathrm{P} \exp \left[\int_{0}^{2 \pi} J_{\sigma}^{g} d \sigma\right] \exp \left(-2 \pi w n_{12}\right) \tilde{g}(\tau, 0) .
$$

Here $\tilde{g}(\tau, \sigma)$ is parametrized as in $(3.15)$ with the undeformed coordinates $\left(\tilde{x}^{0}, \tilde{r}, \tilde{\theta}, \tilde{x}^{3}\right)$.

\section{General arguments}

In this section, let us show that the anticipated form of Lax pair in section 3.2 works for arbitrary classical $r$-matrices of the class (a) satisfying the CYBE. Then we consider a general twisted boundary condition for the class (a)-1. Finally, we argue a general expression of Lax pair for the mCYBE case.

\subsection{Proof of the anticipated Lax pair}

We show that the flatness condition of the anticipated Lax pair (3.2) is equivalent to the equations of motion and confirm that the Lax pair (3.2) works well for all classical $r$-matrices that are solutions of the homogeneous CYBE.

It is convenient to rewrite the Lax pair (3.2) as

$$
\mathcal{L}_{ \pm}=J_{ \pm}^{n}+\lambda^{ \pm 1} J_{ \pm}^{p}, \quad J_{ \pm}^{p} \equiv J_{ \pm}^{\mu} p_{\mu}, \quad J_{ \pm}^{n} \equiv J_{ \pm}^{\mu \nu} n_{\mu \nu} .
$$


The zero-curvature condition of the Lax pair (3.2) can also be rewritten as

$$
\begin{aligned}
0 & =\partial_{+} \mathcal{L}_{-}-\partial_{-} \mathcal{L}_{+}+\left[\mathcal{L}_{+}, \mathcal{L}_{-}\right] \\
& =\lambda\left(-\partial_{-} J_{+}^{p}+\left[J_{+}^{p}, J_{-}^{n}\right]\right)+\frac{1}{\lambda}\left(\partial_{+} J_{-}^{p}+\left[J_{+}^{n}, J_{-}^{p}\right]\right)+\partial_{+} J_{-}^{n}-\partial_{-} J_{+}^{n}+\left[J_{+}^{n}, J_{-}^{n}\right] .
\end{aligned}
$$

The coefficients of $\lambda^{ \pm}$and $\lambda^{0}$ should vanish respectively. Hence the zero-curvature condition is equivalent to the following set of three equations:

$$
\begin{aligned}
\partial_{-} J_{+}^{p}-\left[J_{+}^{p}, J_{-}^{n}\right] & =0, \quad \partial_{+} J_{-}^{p}+\left[J_{+}^{n}, J_{-}^{p}\right]=0, \\
\partial_{+} J_{-}^{n}-\partial_{-} J_{+}^{n}+\left[J_{+}^{n}, J_{-}^{n}\right] & =0 .
\end{aligned}
$$

Then the remaining task is to confirm that the three equations in (4.3) are equivalent to the equations of motion of the deformed system with classical $r$-matrices of the class (a) and the zero-curvature condition for $A_{\mu}$.

Equations of motion. Taking a variation of the classical action (2.1), one can derive the equations of motion ${ }^{3}$

$$
\operatorname{Tr}\left[\mathcal{E} p_{\mu}\right]=0, \quad \mathcal{E} \equiv \partial_{+} P\left(J_{-}\right)+\partial_{-} P\left(J_{+}\right)+\left[J_{+}, P\left(J_{-}\right)\right]+\left[J_{-}, P\left(J_{+}\right)\right] .
$$

Then the equations in (4.4) can be rewritten $\operatorname{as}^{4}$

$$
\operatorname{Tr}\left[\left(\partial_{+} J_{-}^{\rho} \gamma_{\rho}+\partial_{-} J_{+}^{\rho} \gamma_{\rho}+\left[J_{+}^{\rho} p_{\rho}+J_{+}^{\rho \sigma} n_{\rho \sigma}, J_{-}^{\lambda} \gamma_{\lambda}\right]+\left[J_{-}^{\rho} p_{\rho}+J_{-}^{\rho \sigma} n_{\rho \sigma}, J_{+}^{\rho} \gamma_{\rho}\right]\right) p_{\mu}\right]=0 .
$$

In the third and fourth terms, the commutator $\left[\gamma_{\mu}, p_{\nu}\right]\left(=2 n_{\mu \nu}+\eta_{\mu \nu} \gamma_{5}\right)$ can be dropped off because the generators given by this commutator $\left(n_{\mu \nu}\right.$ and $\left.\gamma_{5}\right)$ vanish after taking trace with $p_{\mu}$. For the remaining terms, $\gamma_{\mu}$ can be replaced by $p_{\mu}$ because the commutation relations $\left[\gamma_{\mu}, n_{\nu \rho}\right]$ and $\left[p_{\mu}, n_{\nu \rho}\right]$ take the same form. As a result, the equations of motion in (4.4) are equivalent to the following equations:

$$
\tilde{\mathcal{E}} \equiv \partial_{+} J_{-}^{p}+\partial_{-} J_{+}^{p}+\left[J_{+}^{n}, J_{-}^{p}\right]+\left[J_{-}^{n}, J_{+}^{p}\right]=0 .
$$

Zero-curvature condition. One can rewrite the zero-curvature condition for $A_{ \pm}$in terms of $J_{ \pm}$like

$$
\begin{aligned}
0 & =\mathcal{Z}=\partial_{+} A_{-}-\partial_{-} A_{+}+\left[A_{+}, A_{-}\right] \\
& =\partial_{+} J_{-}-\partial_{-} J_{+}+\left[J_{+}, J_{-}\right]+2 \eta R_{g}(\mathcal{E})+4 \eta^{2} \mathrm{YBE}_{R g}\left(P\left(J_{+}\right), P\left(J_{-}\right)\right),
\end{aligned}
$$

through the relation $A_{ \pm}=\left(1 \mp 2 \eta R_{g} \circ P\right) J_{ \pm}$. Here the symbol $\mathrm{YBE}_{R g}$ in the fifth term is defined as

$$
\mathrm{YBE}_{R g}(X, Y) \equiv\left[R_{g}(X), R_{g}(Y)\right]-R_{g}\left(\left[R_{g}(X), Y\right]+\left[X, R_{g}(Y)\right]\right) .
$$

We are now considering the homogeneous CYBE case, hence the fifth term vanishes.

\footnotetext{
${ }^{3}$ The derivation of (4.4) is given in appendix A of [5].

${ }^{4}$ Note that $J$ can be expanded with $p_{\mu}$ and $n_{\mu \nu}$ within the class (a).
} 
Then, with the help of the relation

$$
R_{g}(\mathcal{E})=R_{g}(\tilde{\mathcal{E}})
$$

the fourth term in the last line of $(4.6)$ can be rewritten as $2 \eta R_{g}(\tilde{\mathcal{E}})$. Thus the last line of (4.6) is recast into

$$
0=\mathcal{Z}=\partial_{+} J_{-}-\partial_{-} J_{+}+\left[J_{+}, J_{-}\right]+2 \eta R_{g}(\tilde{\mathcal{E}}) .
$$

This equality can be decomposed into the $\mathfrak{i s o}(1,3) / \mathfrak{s o}(1,3)$ and $\mathfrak{s o}(1,3)$ components like

$$
\begin{aligned}
0 & =\mathcal{Z}^{p} \equiv \partial_{+} J_{-}^{p}-\partial_{-} J_{+}^{p}+\left[J_{+}^{p}, J_{-}^{n}\right]+\left[J_{+}^{n}, J_{-}^{p}\right]+2 \eta P\left(R_{g}(\tilde{\mathcal{E}})\right), \\
0 & =\mathcal{Z}^{n} \equiv \partial_{+} J_{-}^{n}-\partial_{-} J_{+}^{n}+\left[J_{+}^{n}, J_{-}^{n}\right]+2 \eta P_{0}\left(R_{g}(\tilde{\mathcal{E}})\right) .
\end{aligned}
$$

Note that $P_{0}\left(R_{g}(\tilde{\mathcal{E}})\right)$ and $P\left(R_{g}(\tilde{\mathcal{E}})\right)$ vanish because $\tilde{\mathcal{E}}=0$, and this decomposition is based on the assumption that classical $r$-matrices are limited to the class (a).

Equivalence. Now we have prepared to check the equivalence between $\tilde{\mathcal{E}}=\mathcal{Z}^{p}=\mathcal{Z}^{n}=0$ and the equations in (4.3). The manifest correspondence is summarized below:

$$
\left\{\begin{array}{l}
\tilde{\mathcal{E}}+\mathcal{Z}^{p}=0 \\
\tilde{\mathcal{E}}-\mathcal{Z}^{p}=0 \\
\mathcal{Z}^{n}=0
\end{array} \quad \Longleftrightarrow \quad \text { three equations in }(4.3)\right.
$$

Thus we have shown that the zero-curvature condition of the Lax pair (3.2) is equivalent to the equations of motion of the deformed system with classical $r$-matrices of the class (a) satisfying the homogeneous CYBE.

\subsection{Twisted boundary condition}

Let us next consider the general form of twisted boundary condition (3.21), which was anticipated in section 3.3, in the case of abelian classical $r$-matrices [class (a)-1].

Let us parametrize a group element $g$ as

$$
\begin{aligned}
g & =g_{A} g_{X} \\
g_{A} & =\exp \left(x^{a} a+x^{b} b\right), \quad g_{X}=\prod_{i} \exp \left(y^{i} X_{i}\right) \quad\left[a, b, X_{i} \in \mathfrak{i s o}(1,3)\right],
\end{aligned}
$$

and suppose that a classical $r$-matrix composed of the generators $a$ and $b$ which appear in the definition of $g_{A}$

$$
r=a \wedge b, \quad[a, b]=0 .
$$

Note here that this parameterization (4.11) is assumed to be equivalent to the standard one (B.1) via a gauge transformation like

$$
g(\mathrm{~B} .1) \rightarrow g(\mathrm{~B} .1) h, \quad h \in \mathfrak{s o}(1,3) .
$$

This parameterization ${ }^{5}$ enables us to realize manifestly the translational invariance of the current $A_{\alpha}$ in the $x^{a}$ and $x^{b}$ directions. It is also easy to figure out a deformed part as a replacement law. Therefore we will adopt the parameterization (4.11) to argue a general form of twisted boundary condition.

\footnotetext{
${ }^{5}$ This parametrization has already been utilized in (3.15) and will be used also in section 5 .
} 
Replacement law. Then the left-invariant one-form $A_{\alpha}=g^{-1} \partial_{\alpha} g$ is rewritten as

$$
A_{\alpha}=\partial_{\alpha} x^{a} g_{X}^{-1} a g_{X}+\partial_{\alpha} x^{b} g_{X}^{-1} b g_{X}+g_{X}^{-1} \partial_{\alpha} g_{X},
$$

and the deformed current $J_{ \pm}$is evaluated as

$$
\begin{aligned}
J_{ \pm}= & A_{ \pm} \pm 2 \eta R_{g} \circ P\left(J_{ \pm}\right) \\
= & \left(\partial_{ \pm} x^{a} \pm 2 \eta \operatorname{Tr}\left[g_{X}^{-1} b g_{X} P\left(J_{ \pm}\right)\right]\right) g_{X}^{-1} a g_{X} \\
& +\left(\partial_{ \pm} x^{b} \mp 2 \eta \operatorname{Tr}\left[g_{X}^{-1} a g_{X} P\left(J_{ \pm}\right)\right]\right) g_{X}^{-1} b g_{X}+g_{X}^{-1} \partial_{ \pm} g_{X}
\end{aligned}
$$

Then, comparing (4.14) with (4.15), one can read off the replacement law:

$$
\begin{aligned}
& \partial_{ \pm} x^{a} \quad \rightarrow \partial_{ \pm} x^{a} \pm 2 \eta \operatorname{Tr}\left[g_{X}^{-1} b g_{X} P\left(J_{ \pm}\right)\right] \\
& \partial_{ \pm} x^{b} \rightarrow \partial_{ \pm} x^{b} \mp 2 \eta \operatorname{Tr}\left[g_{X}^{-1} a g_{X} P\left(J_{ \pm}\right)\right] .
\end{aligned}
$$

Twisted boundary condition. First of all, we show that the deformed action is invariant under the translations along the $x^{a}$ and $x^{b}$ directions. Then the second terms in the r.h.s. of (4.16) correspond to the Noether currents associated with these translations.

Let us consider a variation of the deformed action associated with an infinitesimal translation of $x^{a}$ and $x^{b}$

$$
x^{a} \rightarrow x^{a}+\delta x^{a}, \quad x^{b} \rightarrow x^{b}+\delta x^{b} .
$$

The associated variations of $g$ and $A_{ \pm}$are given by

$$
\delta g=\epsilon g, \quad \delta A_{ \pm}=g^{-1}\left(\partial_{ \pm} \epsilon\right) g .
$$

Here an infinitesimal quantity $\epsilon$ is defined as

$$
\epsilon \equiv \delta x^{a} a+\delta x^{b} b .
$$

Then the variation of the deformed action is evaluated as

$$
\begin{aligned}
\delta S & =-\int d^{2} \sigma \gamma^{\alpha \beta} \operatorname{Tr}\left[\partial_{\alpha} \epsilon\left(g P\left(J_{\beta}\right) g^{-1}\right)\right] \\
& =-\int d^{2} \sigma \gamma^{\alpha \beta}\left(\partial_{\alpha} \delta x^{a} \operatorname{Tr}\left[g_{X}^{-1} a g_{X} P\left(J_{\beta}\right)\right]+\partial_{\alpha} \delta x^{b} \operatorname{Tr}\left[g_{X}^{-1} b g_{X} P\left(J_{\beta}\right)\right]\right),
\end{aligned}
$$

by using the following formula in taking a variation of $R_{g}$,

$$
\begin{aligned}
\delta R_{g}(X) & =R_{g}(\delta X)+\left[R_{g}(X), g^{-1} \epsilon g\right]-R_{g}\left(\left[X, g^{-1} \epsilon g\right]\right) \\
& =R_{g}(\delta X) .
\end{aligned}
$$

Note that the last two terms of the first line vanish in the case of abelian $r$-matrices. From (4.19), the deformed action is invariant under the translation along the $x^{a}$ and $x^{b}$ directions. Then the associated Noether currents are given by

$$
P_{a}^{\alpha}=-\gamma^{\alpha \beta} \operatorname{Tr}\left[g_{X}^{-1} a g_{X} P\left(J_{\beta}\right)\right], \quad P_{b}^{\alpha}=-\gamma^{\alpha \beta} \operatorname{Tr}\left[g_{X}^{-1} b g_{X} P\left(J_{\beta}\right)\right] .
$$


One can rewrite the replacement law with these Noether currents. In particular, the $\sigma$-components are given by

$$
\partial_{\sigma} \tilde{x}^{a}=\partial_{\sigma} x^{a}+2 \eta P_{b}^{\tau}, \quad \partial_{\sigma} \tilde{x}^{b}=\partial_{\sigma} x^{b}-2 \eta P_{a}^{\tau} .
$$

Then, by integrating the equations in (4.22), twisted boundary conditions are obtained as

$$
\begin{aligned}
& \tilde{x}^{a}(\tau, \sigma=2 \pi)=\tilde{x}^{a}(\tau, \sigma=0)+2 \eta P_{b}, \\
& \tilde{x}^{b}(\tau, \sigma=2 \pi)=\tilde{x}^{b}(\tau, \sigma=0)-2 \eta P_{a} .
\end{aligned}
$$

Here $P^{a}$ and $P^{b}$ are the Noether charges defined as follows:

$$
P_{a} \equiv \int_{0}^{2 \pi} d \sigma P_{a}^{\tau}, \quad P_{b} \equiv \int_{0}^{2 \pi} d \sigma P_{b}^{\tau} .
$$

These boundary conditions can be recast into a simple form,

$$
\tilde{g}(\tau, \sigma=2 \pi)=\exp \left[2 \eta \int_{0}^{2 \pi} d \sigma\left(P_{b}^{\tau} a-P_{a}^{\tau} b\right)\right] \tilde{g}(\tau, \sigma=0),
$$

with a coset representative $\tilde{g}$ for the undeformed background. Then the twist factor can be rewritten as follows:

$$
\begin{aligned}
2 \eta\left(P_{b}^{\tau} a-P_{a}^{\tau} b\right) & =2 \eta\left(\operatorname{Tr}\left[g_{X}^{-1} b g_{X} P\left(J_{\tau}\right)\right] a-\operatorname{Tr}\left[g_{X}^{-1} a g_{X} P\left(J_{\tau}\right)\right] b\right) \\
& =2 \eta g R_{g}\left(J_{\tau}\right) g^{-1}=g\left(J_{\sigma}-A_{\sigma}\right) g^{-1} \\
& \equiv J_{\sigma}^{g}
\end{aligned}
$$

With this relation, the twisted boundary condition can be rewritten into a general form,

$$
\tilde{g}(\tau, \sigma=2 \pi)=\exp \left[\int_{0}^{2 \pi} d \sigma J_{\sigma}^{g}\right] \tilde{g}(\tau, \sigma=0),
$$

which is now independent of $x^{a}$ and $x^{b}$.

\subsection{The case of $\mathrm{mCYBE}$}

So far, we have concentrated on the homogeneous CYBE case. Now it is easy to generalize the Lax pair to the mCYBE case. All we have to do is a slight modification to add an appropriate term to the previous Lax pair (4.1) as follows:

$$
\mathcal{L}_{ \pm}=J_{ \pm}^{n}+\lambda^{ \pm 1}\left(J_{ \pm}^{p}+\omega \eta^{2} J_{ \pm}^{\tilde{k}}\right), \quad J_{ \pm}^{\tilde{k}} \equiv J_{ \pm}^{\mu} k_{\mu} .
$$

Here $\omega$ is a constant that appears on the r.h.s. of the mCYBE in (2.4).

Note here that the expression (4.28) contains the case of classical $r$-matrices for the general $\kappa$-deformations of $4 \mathrm{D}$ Poincaré algebra [40]. The proof that the Lax pair (4.28) works well is quite similar to the one in [40]. Hence we will not try to prove it here. 


\section{Other examples}

In this section, we will present explicit forms of Lax pairs for twisted Minkowski spacetimes associated with classical $r$-matrices of the class (a)-1. We also derive twisted boundary conditions on the undeformed coordinates by following a simple replacement rule [17, 23, 30]. It is easy to rewrite the twisted boundary conditions into simple forms by adopting appropriate representatives of group element. Hence we will work with a suitable representative for each of classical $r$-matrices, rather than the standard one (B.1).

In the following, we will present explicit forms of Lax pairs and twisted boundary conditions for three cases, 1) pp-wave backgrounds, 2) the Hashimoto-Sethi background and 3) the Spradlin-Takayanagi-Volovich background.

\section{1 pp-wave backgrounds}

We consider an abelian classical $r$-matrix

$$
r=\frac{1}{2 \sqrt{2}}\left(p_{0}-p_{3}\right) \wedge n_{12} .
$$

This classical $r$-matrix is associated with pp-wave backgrounds [46] .

The deformed background. A convenient representative of group element is given by

$$
\begin{aligned}
g(\tau, \sigma) & =\exp \left[x^{-} \frac{\left(p_{0}-p_{3}\right)}{\sqrt{2}}-\theta n_{12}\right] \exp \left[x^{+} \frac{\left(p_{0}+p_{3}\right)}{\sqrt{2}}+r p_{1}\right] \\
& =\exp \left[x^{+} \frac{\left(p_{0}+p_{3}\right)}{\sqrt{2}}+x^{-} \frac{\left(p_{0}-p_{3}\right)}{\sqrt{2}}+r \cos \theta p_{1}+r \sin \theta p_{2}\right] \exp \left[-\theta n_{12}\right] .
\end{aligned}
$$

Here $x^{+}$and $x^{-}$are the light-cone coordinates defined as

$$
x^{+} \equiv \frac{x^{0}+x^{3}}{\sqrt{2}}, \quad x^{-} \equiv \frac{x^{0}-x^{3}}{\sqrt{2}} .
$$

The representation (5.2) is equivalent to the standard one (B.1) via a gauge transformation.

Now the undeformed current $A=g^{-1} d g$ is given by

$$
A_{ \pm}=\partial_{ \pm} x^{+} \frac{p_{0}+p_{3}}{\sqrt{2}}+\partial_{ \pm} x^{-} \frac{p_{0}-p_{3}}{\sqrt{2}}+\partial_{ \pm} r p_{1}+\partial_{ \pm} \theta\left[r p_{2}-n_{12}\right],
$$

and it leads to the standard metric of 4D Minkowski spacetime:

$$
d s^{2}=-2 d x^{+} d x^{-}+d r^{2}+r^{2} d \theta^{2} .
$$

Then the deformed current $J_{ \pm}$is expanded like

$$
\begin{aligned}
J_{ \pm}= & \partial_{ \pm} x^{+} \frac{p_{0}+p_{3}}{\sqrt{2}}+\left(\partial_{ \pm} x^{-} \mp \eta r^{2} \partial_{ \pm} \theta+\eta^{2} r^{2} \partial_{ \pm} x^{+}\right) \frac{p_{0}-p_{3}}{\sqrt{2}} \\
& +\partial_{ \pm} r p_{1}+\left(\partial_{ \pm} \theta \mp \eta \partial_{ \pm} x^{+}\right)\left[r p_{2}-n_{12}\right] .
\end{aligned}
$$

With this deformed current, the resulting metric and NS-NS two-form are given by

$$
\begin{aligned}
d s^{2} & =-2 d x^{+} d x^{-}-\eta^{2} r^{2}\left(d x^{+}\right)^{2}+d r^{2}+r^{2} d \theta^{2}, \\
B & =\eta r^{2} d x^{+} \wedge d \theta .
\end{aligned}
$$


Lax pair. The associated Lax pair is given by

$$
\begin{aligned}
\mathcal{L}_{ \pm}=-\left(\partial_{ \pm} \theta \mp \eta \partial_{ \pm} x^{+}\right) n_{12}+\lambda^{ \pm 1}\left[\partial_{ \pm} r p_{1}+r\left(\partial_{ \pm} \theta \mp \eta \partial_{ \pm} x^{+}\right) p_{2}\right. \\
\left.+\partial_{ \pm} x^{+} \frac{p_{0}+p_{3}}{\sqrt{2}}+\left(\partial_{ \pm} x^{-} \mp \eta r^{2} \partial_{ \pm} \theta+\eta^{2} r^{2} \partial_{ \pm} x^{+}\right) \frac{p_{0}-p_{3}}{\sqrt{2}}\right] .
\end{aligned}
$$

By comparing the deformed current with the undeformed one, one can identify the following replacement rule:

$$
\begin{aligned}
& \partial_{ \pm} x^{-} \rightarrow \partial_{ \pm} x^{-} \mp \eta r^{2} \partial_{ \pm} \theta+\eta^{2} r^{2} \partial_{ \pm} x^{+} \\
& \partial_{ \pm} \theta \rightarrow \partial_{ \pm} \theta \mp \eta \partial_{ \pm} x^{+} .
\end{aligned}
$$

Note that the deformed Lax pair can also be reproduced by applying the replacement rule (5.8) to the undeformed Lax pair (3.11) in which U(1)-directions are manifest.

Twisted boundary condition. The pp-wave background with usual boundary conditions can be seen as as the undeformed Minkowski space with a twisted boundary conditions, as in the Melvin case. In terms of the undeformed group element $\tilde{g}(\tau, \sigma)$, the twisted boundary condition is given by

$$
\tilde{g}(\tau, 2 \pi)=\mathrm{P} \exp \left[\int_{0}^{2 \pi} d \sigma J_{\sigma}^{g}\right] \exp \left[2 \pi m\left(-n_{12}\right)\right] \tilde{g}(\tau, 0)
$$

where the integer $m$ is a winding number for $\theta$. Here the gauge-transformed current $J_{\alpha}^{g}=g J_{\alpha} g^{-1}+g \partial_{\alpha} g^{-1}$ takes the following form:

$$
\begin{aligned}
J_{\sigma}^{g} & =-\eta r^{2}\left(\dot{\theta}-\eta x^{\prime+}\right) \frac{p_{0}-p_{3}}{\sqrt{2}}+\eta \dot{x}^{+} n_{12} \\
& =-\eta \mathcal{J}_{\theta}^{\tau} \frac{p_{0}-p_{3}}{\sqrt{2}}-\eta P_{-}^{\tau} n_{12} .
\end{aligned}
$$

More concretely, the twisted boundary condition can be rewritten as

$$
\begin{aligned}
& \tilde{x}^{-}(\tau, 2 \pi)=\tilde{x}^{-}(\tau, 0)-\eta \mathcal{J}_{\theta}, \\
& \tilde{\theta}^{-}(\tau, 2 \pi)=\tilde{\theta}^{-}(\tau, 0)+\eta P_{-}+2 \pi m .
\end{aligned}
$$

Here $\mathcal{J}_{\theta}$ and $P_{-}$are Noether charges for the invariance under the rotation and translation along the $\theta$ and $x^{-}$directions, respectively.

\subsection{Hashimoto-Sethi background}

Let us next consider a classical $r$-matrix,

$$
r=-\frac{1}{2 \sqrt{2}} p_{2} \wedge\left(n_{01}+n_{13}\right) .
$$

This $r$-matrix leads to a time-dependent background obtained via a Melvin Null twist of Minkowski spacetime [47]. 
The deformed background. A useful group parametrization is given by

$$
\begin{aligned}
g(\tau, \sigma)= & \exp \left[z p_{2}+y \frac{n_{01}+n_{13}}{\sqrt{2}}\right] \exp \left[y^{+} \frac{p_{0}+p_{3}}{\sqrt{2}}+y^{-} \frac{p_{0}-p_{3}}{\sqrt{2}}\right] \\
= & \exp \left[y^{+} y p_{1}+z p_{2}+y^{+} \frac{p_{0}+p_{3}}{\sqrt{2}}+\left(y^{-}+\frac{1}{2} y^{+} y^{2}\right) \frac{p_{0}-p_{3}}{\sqrt{2}}\right] \\
& \times \exp \left[y \frac{n_{01}+n_{13}}{\sqrt{2}}\right] .
\end{aligned}
$$

Here we have performed the following coordinate transformation:

$$
x^{1}=y^{+} y, \quad x^{2}=z, \quad x^{+}=y^{+}, \quad x^{-}=y^{-}+\frac{1}{2} y^{+} y^{2} .
$$

Note that this coordinate transformation is singular at $y^{+}=0$. Now the undeformed current $A=g^{-1} d g$ and the associated singular metric are given by, respectively,

$$
\begin{aligned}
& A_{ \pm}=\partial_{ \pm} y^{+} \frac{p_{0}+p_{3}}{\sqrt{2}}+\partial_{ \pm} y^{-} \frac{p_{0}-p_{3}}{\sqrt{2}}+\partial_{ \pm} y\left[y^{+} p_{1}+\frac{n_{13}+n_{01}}{\sqrt{2}}\right]+\partial_{ \pm} z p_{2} \\
& d s^{2}=-2 d y^{+} d y^{-}+\left(y^{+}\right)^{2} d y^{2}+d z^{2}
\end{aligned}
$$

Then the deformed current $J_{ \pm}$is given by

$$
\begin{aligned}
J_{ \pm}= & \partial_{ \pm} y^{+} \frac{p_{0}+p_{3}}{\sqrt{2}}+\partial_{ \pm} y^{-} \frac{p_{0}-p_{3}}{\sqrt{2}} \\
& +G_{\mathrm{HS}}\left(\partial_{ \pm} y \pm \eta \partial_{ \pm} z\right)\left[y^{+} p_{1}+\frac{n_{13}+n_{01}}{\sqrt{2}}\right]+G_{\mathrm{HS}}\left[\partial_{ \pm} z \mp \eta\left(y^{+}\right)^{2} \partial_{ \pm} y\right] p_{2},
\end{aligned}
$$

with a scalar function $G_{\mathrm{HS}}$ defined as

$$
G_{\mathrm{HS}}^{-1} \equiv 1+\eta^{2}\left(y^{+}\right)^{2}
$$

As a result, the deformed metric and NS-NS two-form are obtained as

$$
\begin{aligned}
d s^{2} & =-2 d y^{+} d y^{-}+G_{\mathrm{HS}}\left[\left(y^{+}\right)^{2} d y^{2}+d z^{2}\right], \\
B & =\eta\left(y^{+}\right)^{2} G_{\mathrm{HS}} d y \wedge d z .
\end{aligned}
$$

This background is also realized by performing a TsT-transformation with the $y$ and $z$ in the undeformed background (5.17) under the identification $[47,56]$

$$
g(\tau, \sigma) \sim g_{0} g(\tau, \sigma), \quad g_{0}=\exp \left[2 \pi\left(p_{2}+\frac{n_{01}+n_{13}}{\sqrt{2}}\right)\right] .
$$

Lax pair. The resulting Lax pair for the Hashimoto-Sethi background is given by

$$
\begin{aligned}
\mathcal{L}_{ \pm}= & G_{\mathrm{HS}}\left(\partial_{ \pm} y \pm \eta \partial_{ \pm} z\right) \frac{n_{13}+n_{01}}{\sqrt{2}} \\
+\lambda^{ \pm 1}\left[\partial_{ \pm} y+\frac{p_{0}+p_{3}}{\sqrt{2}}+\partial_{ \pm} y^{-} \frac{p_{0}-p_{3}}{\sqrt{2}}\right. & \\
& \left.\quad+G_{\mathrm{HS}} y^{+}\left(\partial_{ \pm} y \pm \eta \partial_{ \pm} z\right) p_{1}+G_{\mathrm{HS}}\left[\partial_{ \pm} z \mp \eta\left(y^{+}\right)^{2} \partial_{ \pm} y\right] p_{2}\right]
\end{aligned}
$$

By comparing $J$ with $A$, one can read off the following rule:

$$
\begin{aligned}
\partial_{ \pm} y & \rightarrow G_{\mathrm{HS}}\left[\partial_{ \pm} y \pm \eta \partial_{ \pm} z\right] \\
\partial_{ \pm} z & \rightarrow G_{\mathrm{HS}}\left[\partial_{ \pm} z \mp \eta\left(y^{+}\right)^{2} \partial_{ \pm} y\right] .
\end{aligned}
$$


Twisted boundary condition. In the present case, the deformed background is equivalent to the undeformed background with the following twisted boundary condition:

$$
\tilde{g}(\tau, 2 \pi)=\mathrm{P} \exp \left[\int_{0}^{2 \pi} d \sigma J_{\sigma}^{g}\right] \exp \left[2 \pi\left(m p_{2}+w \frac{n_{01}+n_{13}}{\sqrt{2}}\right)\right] \tilde{g}(\tau, 0),
$$

where $\tilde{g}(\tau, \sigma)$ is a group element for the undeformed background. The integers $m$ and $w$ are winding numbers defined as

$$
y(\tau, 2 \pi)-y(\tau, 0) \equiv 2 \pi w, \quad z(\tau, 2 \pi)-z(\tau, 0) \equiv 2 \pi m .
$$

The gauge-transformed current $J_{\alpha}^{g}=g J_{\alpha} g^{-1}+g \partial_{\alpha} g^{-1}$ is given by

$$
\begin{aligned}
J_{\sigma}^{g} & =-\eta\left(y^{+}\right)^{2} G_{\mathrm{HS}}\left(\dot{y}+\eta z^{\prime}\right) p_{2}+\eta G_{\mathrm{HS}}\left[\dot{z}-\eta\left(y^{+}\right)^{2} y^{\prime}\right] \frac{n_{01}+n_{13}}{\sqrt{2}} \\
& =-\eta P_{y}^{\tau} p_{2}+\eta P_{z}^{\tau} \frac{n_{01}+n_{13}}{\sqrt{2}} .
\end{aligned}
$$

More concretely, the twisted boundary condition can also be rewritten as

$$
\begin{aligned}
& \tilde{y}(\tau, 2 \pi)=\tilde{y}(\tau, 0)+\eta P_{z}+2 \pi w, \\
& \tilde{z}(\tau, 2 \pi)=\tilde{z}(\tau, 0)-\eta P_{y}+2 \pi m,
\end{aligned}
$$

where $P_{z}$ and $P_{y}$ are conserved charges for the translation invariance along the $z$ and $y$ directions, respectively.

\subsection{Spradlin-Takayanagi-Volovich background}

Finally, we shall consider an abelian classical $r$-matrix composed of two rotation generators,

$$
r=\frac{1}{2} n_{12} \wedge n_{03}
$$

The corresponding background is a time-dependent background [48] as explained below.

The deformed background. Let us parametrize a group element like

$$
\begin{aligned}
g(\tau, \sigma) & =\exp \left[\phi n_{03}-\theta n_{12}\right] \exp \left[t p_{0}+r p_{1}\right] \\
& =\exp \left[t \cosh \phi p_{0}+t \sinh \phi p_{3}+r \cos \theta p_{1}+r \sin \theta p_{2}\right] \times \exp \left[\phi n_{03}-\theta n_{12}\right]
\end{aligned}
$$

Here we have introduced new coordinates $t$ and $\phi$ by performing a coordinate transformation $x^{0}=t \cosh \phi$ and $x^{3}=t \sinh \phi$. Then the undeformed current $A$ is expanded as

$$
A_{ \pm}=\partial_{ \pm} r p_{1}+\partial_{ \pm} t p_{0}+\partial_{ \pm} \theta\left[r p_{2}-n_{12}\right]+\partial_{ \pm} \phi\left[t p_{3}+n_{03}\right] .
$$

As a result, the associated undeformed metric takes the form

$$
d s^{2}=-d t^{2}+t^{2} d \phi^{2}+d r^{2}+r^{2} d \theta^{2} .
$$


Then the deformed current $J_{ \pm}$with the classical $r$-matrix (5.28) is evaluated as

$$
\begin{aligned}
J_{ \pm}= & \partial_{ \pm} r p_{1}+\partial_{ \pm} t p_{0}+G_{\mathrm{STV}}\left(\partial_{ \pm} \theta \mp \eta t^{2} \partial_{ \pm} \phi\right)\left(r p_{2}-n_{12}\right) \\
& +G_{\mathrm{STV}}\left(\partial_{ \pm} \phi \pm \eta r^{2} \partial_{ \pm} \theta\right)\left(t p_{3}+n_{03}\right),
\end{aligned}
$$

where we have introduced a scalar function $G_{\text {STV }}$ defined as

$$
G_{\mathrm{STV}}^{-1} \equiv 1+\eta^{2} r^{2} t^{2}
$$

The resulting deformed background is therefore given by

$$
\begin{aligned}
d s^{2} & =-d t^{2}+d r^{2}+G_{\mathrm{STV}}\left(t^{2} d \phi^{2}+r^{2} d \theta^{2}\right), \\
B & =\eta t^{2} r^{2} G_{\mathrm{STV}} d \phi \wedge d \theta
\end{aligned}
$$

Lax pair. The associated Lax pair with a spectral parameter $\lambda$ is expressed as

$$
\begin{aligned}
\mathcal{L}_{ \pm}= & -G_{\mathrm{STV}}\left(\partial_{ \pm} \theta \mp \eta t^{2} \partial_{ \pm} \phi\right) n_{12}+G_{\mathrm{STV}}\left(\partial_{ \pm} \phi \pm \eta r^{2} \partial_{ \pm} \theta\right) n_{03} \\
& +\lambda^{ \pm 1}\left[\partial_{ \pm} r p_{1}+\partial_{ \pm} t p_{0}+r G_{\mathrm{STV}}\left(\partial_{ \pm} \theta \mp \eta t^{2} \partial_{ \pm} \phi\right) p_{2}+t G_{\mathrm{STV}}\left(\partial_{ \pm} \phi \pm \eta r^{2} \partial_{ \pm} \theta\right) p_{3}\right]
\end{aligned}
$$

Twisted boundary condition. Similarly, by comparing the deformed current with the undeformed one, one can read off the following replacement rule:

$$
\begin{aligned}
\partial_{ \pm} \theta & \rightarrow G_{\mathrm{STV}}\left(\partial_{ \pm} \theta \mp \eta t^{2} \partial_{ \pm} \phi\right), \\
\partial_{ \pm} \phi & \rightarrow G_{\mathrm{STV}}\left(\partial_{ \pm} \phi \pm \eta r^{2} \partial_{ \pm} \theta\right) .
\end{aligned}
$$

This rule implies a twisted boundary condition for the undeformed coordinates,

$$
\tilde{g}(\tau, 2 \pi)=\mathrm{P} \exp \left[\int_{0}^{2 \pi} d \sigma J_{\sigma}^{g}\right] \exp \left[2 \pi\left(m n_{03}-w n_{12}\right)\right] \tilde{g}(\tau, 0) .
$$

Here the integer numbers $w$ and $m$ are winding numbers for the angles $\theta$ and $\phi$. The gauge-transformed current $J_{\alpha}^{g}=g J_{\alpha} g^{-1}+g \partial_{\alpha} g^{-1}$ is given by

$$
\begin{aligned}
J_{\sigma}^{g} & =\eta r^{2} G_{\mathrm{STV}}\left(\dot{\theta}-\eta t^{2} \phi^{\prime}\right) n_{03}+\eta t^{2} G_{\mathrm{STV}}\left(\dot{\phi}+\eta r^{2} \theta^{\prime}\right) n_{12} \\
& =\eta \mathcal{J}_{\theta}^{\tau} n_{03}+\eta \mathcal{J}_{\phi}^{\tau} n_{12} .
\end{aligned}
$$

This expression can also be recast in

$$
\begin{aligned}
\tilde{\theta}(\tau, 2 \pi) & =\tilde{\theta}(\tau, 0)-\eta \mathcal{J}_{\phi}+2 \pi w, \\
\tilde{\phi}(\tau, 2 \pi) & =\tilde{\phi}(\tau, 0)+\eta \mathcal{J}_{\theta}+2 \pi m,
\end{aligned}
$$

where $\mathcal{J}_{\phi}$ and $\mathcal{J}_{\theta}$ are Noether charges for the rotational invariance for the $\phi$ and $\theta$ directions. 


\section{Conclusion and discussion}

In this paper, we have proceeded to study Yang-Baxter deformations of $4 \mathrm{D}$ Minkowski spacetime based on a conformal embedding. In the first place, we have revisited a Melvin background and argued a Lax pair by adopting a simple replacement law invented in [30]. Due to this argument, one can readily deduce a general expression of Lax pair. Then the anticipated Lax pair has been shown to work for arbitrary classical $r$-matrices with Poincaré generators. As other examples, we have presented Lax pairs for pp-wave backgrounds, the Hashimoto-Sethi background, the Spradlin-Takayanagi-Volovich background.

There are a lot of open problems. We have considered only the bosonic sector so far. It would be very interesting to investigate the RR-sector and dilaton by including spacetime fermions (c.f., see [57]). In fact, it is much easier to perform a supercoset construction for Yang-Baxter deformed Minkowski spacetimes, in comparison to the deformed $\mathrm{AdS}_{5} \times \mathrm{S}^{5}$. It is also nice to study non-local charges obtained from the monodromy matrices constructed from the Lax pair derived here. The resulting algebras may be related to the preceding results obtained in [58].

As another direction, it would be of interest to consider Yang-Baxter deformations of other backgrounds such as pp-wave backgrounds and Lifshitz spacetimes [59]. Along this direction, it has been shown that the Nappi-Witten background [46] is Yang-Baxter invariant at the quantum level [60]. Due to the argument in [61], Lifshitz spacetimes would be invariant as well. It is very nice to check whether this anticipation is valid or not.

\section{Acknowledgments}

We are very grateful to Andrzej Borowiec, Jerzy Lukierski, Takuya Matsumoto, Domenico Orlando, Susanne Reffert and Stijn van Tongeren for useful discussions and collaborations on the previous works. The work of K.Y. is supported by Supporting Program for Interaction-based Initiative Team Studies (SPIRITS) from Kyoto University and by the JSPS Grant-in-Aid for Scientific Research (C) No.15K05051. This work is also supported in part by the JSPS Japan-Russia Research Cooperative Program and the JSPS JapanHungary Research Cooperative Program.

\section{A Notation and convention}

We will summarize here our notation and convention of the $\mathfrak{s o}(2,4)$ generators.

In this paper, we use the following basis of $\mathfrak{s u}(2,2) \simeq \mathfrak{s o}(2,4)$ :

$$
\mathfrak{s u}(2,2)=\operatorname{span}_{\mathbb{R}}\left\{\gamma_{\mu}, \gamma_{5}, n_{\mu \nu}=\frac{1}{4}\left[\gamma_{\mu}, \gamma_{\nu}\right], n_{\mu 5}=\frac{1}{4}\left[\gamma_{\mu}, \gamma_{5}\right] \mid \mu, \nu=0,1,2,3\right\} .
$$

Here $\gamma_{\mu}$ 's are gamma matrices satisfying the Dirac algebra:

$$
\left\{\gamma_{\mu}, \gamma_{\nu}\right\}=2 \eta_{\mu \nu}
$$


Here $\eta_{\mu \nu}$ is the standard Minkowski metric with mostly plus. It is convenient to adopt the following matrix realization of $\gamma_{\mu}$ 's,

$$
\begin{array}{rlr}
\gamma_{1} & =\left(\begin{array}{cccc}
0 & 0 & 0 & -1 \\
0 & 0 & 1 & 0 \\
0 & 1 & 0 & 0 \\
-1 & 0 & 0 & 0
\end{array}\right), \\
\gamma_{3} & =\left(\begin{array}{cccc}
0 & 0 & 1 & 0 \\
0 & 0 & 0 & 1 \\
1 & 0 & 0 & 0 \\
0 & 1 & 0 & 0
\end{array}\right), \\
\gamma_{5}=i \gamma_{1} \gamma_{2} \gamma_{3} \gamma_{0} & =\left(\begin{array}{cccc}
1 & 0 & 0 & 0 \\
0 & 1 & 0 & 0 \\
0 & 0 & -1 & 0 \\
0 & 0 & 0 & -1
\end{array}\right)
\end{array}
$$

It is also helpful to use the conformal basis for $\mathfrak{s o}(2,4)$ :

$$
\mathfrak{s o}(2,4)=\operatorname{span}_{\mathbb{R}}\left\{p_{\mu}, n_{\mu \nu}, \hat{d}, k_{\mu} \mid \mu, \nu=0,1,2,3\right\} .
$$

Here the translation generators $p_{\mu}$, the Lorentz rotation generators $n_{\mu \nu}$, the dilatation $\hat{d}$ and the special conformal generators $k_{\mu}$ are represented by, respectively,

$$
p_{\mu} \equiv \frac{1}{2}\left(\gamma_{\mu}-2 n_{\mu 5}\right), \quad \hat{d} \equiv \frac{1}{2} \gamma_{5}, \quad k_{\mu} \equiv \frac{1}{2}\left(\gamma_{\mu}+2 n_{\mu 5}\right) .
$$

The non-vanishing commutation relations are given by

$$
\begin{aligned}
{\left[p_{\mu}, k_{\nu}\right] } & =2\left(n_{\mu \nu}+\eta_{\mu \nu} \hat{d}\right), \quad\left[\hat{d}, p_{\mu}\right]=p_{\mu}, \quad\left[\hat{d}, k_{\mu}\right]=-k_{\mu}, \\
{\left[p_{\mu}, n_{\nu \rho}\right] } & =\eta_{\mu \nu} p_{\rho}-\eta_{\mu \rho} p_{\nu}, \quad\left[k_{\mu}, n_{\nu \rho}\right]=\eta_{\mu \nu} k_{\rho}-\eta_{\mu \rho} k_{\nu}, \\
{\left[n_{\mu \nu}, n_{\rho \sigma}\right] } & =\eta_{\mu \sigma} n_{\nu \rho}+\eta_{\nu \rho} n_{\mu \sigma}-\eta_{\mu \rho} n_{\nu \sigma}-\eta_{\nu \sigma} n_{\mu \rho} .
\end{aligned}
$$

\section{B A conformal embedding of 4D Minkowski spacetime}

To introduce Yang-Baxter deformations of 4D Minkowski spacetime, it is necessary to perform a coset construction of flat space. However there is an obstacle that the Killing form on the standard coset $\operatorname{ISO}(1,3) / \mathrm{SO}(1,3)$ is degenerate. Hence, in order to avoid this issue, we will embed this coset into a conformal group $\mathrm{SO}(2,4)$, as explained below.

A representative of group element $g$ is given by

$$
g=\exp \left[p_{0} x^{0}+p_{1} x^{1}+p_{2} x^{2}+p_{3} x^{3}\right]
$$

Note that this parametrization may be interpreted as a slice of Poincaré $\mathrm{AdS}_{5}$, in which the representative is given by $g=\exp \left[x^{\mu} p_{\mu}\right] \exp [\hat{d} \log z]$ with the AdS radial direcrion $z$.

The left-invariant one-form $A=g^{-1} d g$ can be expanded as

$$
A \equiv e^{\mu} p_{\mu}, \quad e^{\mu}=d x^{\mu} .
$$


Then, in terms of the vierbeins $e^{\mu}$, the metric of Minkowski spacetime is given by

$$
d s^{2}=\eta_{\mu \nu} e^{\mu} e^{\nu}=\eta_{\mu \nu} d x^{\mu} d x^{\nu} .
$$

By using the relations

$$
e^{\mu}=\frac{1}{2} \operatorname{Tr}\left(\gamma^{\mu} A\right),
$$

the metric (B.3) can be recast into the following form:

$$
d s^{2}=\eta_{\mu \nu} e^{\mu} e^{\nu}=\operatorname{Tr}(A P(A)) .
$$

Here we have introduced a projection operator defined as

$$
P(x) \equiv \frac{1}{4}\left[-\gamma_{0} \operatorname{Tr}\left(\gamma_{0} x\right)+\sum_{i=1}^{3} \gamma_{i} \operatorname{Tr}\left(\gamma_{i} x\right)\right] .
$$

Note that $\gamma^{\mu}$ 's contained in (B.6) are elements of 4D conformal algebra $\mathfrak{s o}(2,4)$ rather than 4D Poincaré algebra iso $(1,3)$. Thus the projection (B.6) implies that our coset construction of $4 \mathrm{D}$ Minkowski spacetime is realized as a slice of Poincaré $\mathrm{AdS}_{5}$.

As a side note, it is straightforward to generalize the projection operator to the general symmetric two-form [46, 61]. Due to this observation, Yang-Baxter deformations can be applied to the Schrödinger and Lifshitz cosets argued in [61]. For a recent progress along this line, see the work [60] in which Yang-Baxter deformations of the Nappi-Witten model have been studied.

Open Access. This article is distributed under the terms of the Creative Commons Attribution License (CC-BY 4.0), which permits any use, distribution and reproduction in any medium, provided the original author(s) and source are credited.

\section{References}

[1] C. Klimčík, Yang-Baxter $\sigma$-models and dS/AdS T duality, JHEP 12 (2002) 051 [hep-th/0210095] [INSPIRE].

[2] C. Klimčík, On integrability of the Yang-Baxter $\sigma$-model, J. Math. Phys. 50 (2009) 043508 [arXiv: 0802.3518] [INSPIRE].

[3] C. Klimčík, Integrability of the bi-Yang-Baxter $\sigma$-model, Lett. Math. Phys. 104 (2014) 1095 [arXiv: 1402.2105] [INSPIRE].

[4] F. Delduc, M. Magro and B. Vicedo, On classical q-deformations of integrable $\sigma$-models, JHEP 11 (2013) 192 [arXiv: 1308.3581] [INSPIRE].

[5] T. Matsumoto and K. Yoshida, Yang-Baxter $\sigma$-models based on the CYBE, Nucl. Phys. B 893 (2015) 287 [arXiv: 1501.03665] [INSPIRE].

[6] F. Delduc, M. Magro and B. Vicedo, An integrable deformation of the $A d S_{5} \times S^{5}$ superstring action, Phys. Rev. Lett. 112 (2014) 051601 [arXiv:1309.5850] [INSPIRE].

[7] F. Delduc, M. Magro and B. Vicedo, Derivation of the action and symmetries of the q-deformed $A d S_{5} \times S^{5}$ superstring, JHEP 10 (2014) 132 [arXiv:1406.6286] [INSPIRE]. 
[8] G. Arutyunov, R. Borsato and S. Frolov, S-matrix for strings on $\eta$-deformed $A d S_{5} \times S^{5}$, JHEP 04 (2014) 002 [arXiv: 1312.3542] [INSPIRE].

[9] G. Arutyunov, R. Borsato and S. Frolov, Puzzles of $\eta$-deformed $A d S_{5} \times S^{5}$, JHEP 12 (2015) 049 [arXiv: 1507.04239] [INSPIRE].

[10] B. Hoare and A.A. Tseytlin, Type IIB supergravity solution for the T-dual of the $\eta$-deformed $A d S_{5} \times S^{5}$ superstring, JHEP 10 (2015) 060 [arXiv:1508.01150] [INSPIRE].

[11] G. Arutyunov, S. Frolov, B. Hoare, R. Roiban and A.A. Tseytlin, Scale invariance of the eta-deformed $A d S_{5} \times S^{5}$ superstring, T-duality and modified type-II equations, arXiv: 1511.05795 [INSPIRE].

[12] I. Kawaguchi, T. Matsumoto and K. Yoshida, Jordanian deformations of the Ad $S_{5} \times S^{5}$ superstring, JHEP 04 (2014) 153 [arXiv:1401.4855] [INSPIRE].

[13] T. Matsumoto and K. Yoshida, Lunin-Maldacena backgrounds from the classical Yang-Baxter equation - towards the gravity/CYBE correspondence, JHEP 06 (2014) 135 [arXiv: 1404.1838] [INSPIRE].

[14] T. Matsumoto and K. Yoshida, Integrability of classical strings dual for noncommutative gauge theories, JHEP 06 (2014) 163 [arXiv:1404.3657] [INSPIRE].

[15] T. Matsumoto and K. Yoshida, Schrödinger geometries arising from Yang-Baxter deformations, JHEP 04 (2015) 180 [arXiv:1502.00740] [INSPIRE].

[16] O. Lunin and J.M. Maldacena, Deforming field theories with $\mathrm{U}(1) \times \mathrm{U}(1)$ global symmetry and their gravity duals, JHEP 05 (2005) 033 [hep-th/0502086] [INSPIRE].

[17] S. Frolov, Lax pair for strings in Lunin-Maldacena background, JHEP 05 (2005) 069 [hep-th/0503201] [INSPIRE].

[18] A. Hashimoto and N. Itzhaki, Noncommutative Yang-Mills and the AdS/CFT correspondence, Phys. Lett. B 465 (1999) 142 [hep-th/9907166] [INSPIRE].

[19] J.M. Maldacena and J.G. Russo, Large-N limit of noncommutative gauge theories, JHEP 09 (1999) 025 [hep-th/9908134] [INSPIRE].

[20] C.P. Herzog, M. Rangamani and S.F. Ross, Heating up Galilean holography, JHEP 11 (2008) 080 [arXiv: 0807.1099] [INSPIRE].

[21] J. Maldacena, D. Martelli and Y. Tachikawa, Comments on string theory backgrounds with non-relativistic conformal symmetry, JHEP 10 (2008) 072 [arXiv:0807.1100] [INSPIRE].

[22] A. Adams, K. Balasubramanian and J. McGreevy, Hot Spacetimes for Cold Atoms, JHEP 11 (2008) 059 [arXiv:0807.1111] [INSPIRE].

[23] L.F. Alday, G. Arutyunov and S. Frolov, Green-Schwarz strings in TsT-transformed backgrounds, JHEP 06 (2006) 018 [hep-th/0512253] [INSPIRE].

[24] I. Kawaguchi and K. Yoshida, Classical integrability of Schrödinger $\sigma$-models and q-deformed Poincaré symmetry, JHEP 11 (2011) 094 [arXiv: 1109.0872] [INSPIRE].

[25] I. Kawaguchi and K. Yoshida, Exotic symmetry and monodromy equivalence in Schrödinger $\sigma$-models, JHEP 02 (2013) 024 [arXiv: 1209.4147] [INSPIRE].

[26] I. Kawaguchi, T. Matsumoto and K. Yoshida, Schroedinger $\sigma$-models and Jordanian twists, JHEP 08 (2013) 013 [arXiv: 1305.6556] [INSPIRE]. 
[27] S.J. van Tongeren, On classical Yang-Baxter based deformations of the $A d S_{5} \times S^{5}$ superstring, JHEP 06 (2015) 048 [arXiv: 1504.05516] [INSPIRE].

[28] S.J. van Tongeren, Yang-Baxter deformations, AdS/CFT and twist-noncommutative gauge theory, arXiv:1506.01023 [INSPIRE].

[29] B. Vicedo, Deformed integrable $\sigma$-models, classical R-matrices and classical exchange algebra on Drinfel'd doubles, J. Phys. A 48 (2015) 355203 [arXiv:1504.06303] [inSPIRE].

[30] T. Kameyama, H. Kyono, J. Sakamoto and K. Yoshida, Lax pairs on Yang-Baxter deformed backgrounds, JHEP 11 (2015) 043 [arXiv:1509.00173] [INSPIRE].

[31] T. Matsumoto and K. Yoshida, Yang-Baxter deformations and string dualities, JHEP 03 (2015) 137 [arXiv: 1412.3658] [INSPIRE].

[32] I. Kawaguchi, T. Matsumoto and K. Yoshida, A Jordanian deformation of AdS space in type IIB supergravity, JHEP 06 (2014) 146 [arXiv: 1402.6147] [INSPIRE].

[33] P. Basu and L.A. Pando Zayas, Chaos rules out integrability of strings on $A d S_{5} \times T^{1,1}$, Phys. Lett. B 700 (2011) 243 [arXiv: 1103.4107] [INSPIRE].

[34] Y. Asano, D. Kawai, H. Kyono and K. Yoshida, Chaotic strings in a near Penrose limit of $A d S_{5} \times T^{1,1}, J H E P 08(2015) 060$ [arXiv: 1505.07583] [INSPIRE].

[35] A. Catal-Ozer, Lunin-Maldacena deformations with three parameters, JHEP 02 (2006) 026 [hep-th/0512290] [INSPIRE].

[36] P.M. Crichigno, T. Matsumoto and K. Yoshida, Deformations of $T^{1,1}$ as Yang-Baxter $\sigma$-models, JHEP 12 (2014) 085 [arXiv:1406.2249] [INSPIRE].

[37] P.M. Crichigno, T. Matsumoto and K. Yoshida, Towards the gravity/CYBE correspondence beyond integrability - Yang-Baxter deformations of $T^{1,1}$, arXiv:1510.00835 [INSPIRE].

[38] T. Matsumoto and K. Yoshida, Integrable deformations of the $A d S_{5} \times S^{5}$ superstring and the classical Yang-Baxter equation - Towards the gravity/CYBE correspondence -, J. Phys. Conf. Ser. 563 (2014) 012020 [arXiv: 1410.0575] [INSPIRE].

[39] T. Matsumoto, D. Orlando, S. Reffert, J. Sakamoto and K. Yoshida, Yang-Baxter deformations of Minkowski spacetime, JHEP 10 (2015) 185 [arXiv: 1505.04553] [INSPIRE].

[40] A. Borowiec, H. Kyono, J. Lukierski, J. Sakamoto and K. Yoshida, Yang-Baxter $\sigma$-models and Lax pairs arising from $\kappa$-Poincaré $r$-matrices, arXiv:1510.03083 [INSPIRE].

[41] A. Pachol and S.J. van Tongeren, Quantum deformations of the flat space superstring, arXiv: 1510.02389 [INSPIRE].

[42] M.A. Melvin, Pure magnetic and electric geons, Phys. Lett. 8 (1964) 65 [INSPIRE].

[43] G.W. Gibbons and K. Maeda, Black Holes and Membranes in Higher Dimensional Theories with Dilaton Fields, Nucl. Phys. B 298 (1988) 741 [InSPIRE].

[44] A.A. Tseytlin, Melvin solution in string theory, Phys. Lett. B 346 (1995) 55 [hep-th/9411198] [INSPIRE].

[45] A. Hashimoto and K. Thomas, Dualities, twists and gauge theories with non-constant non-commutativity, JHEP 01 (2005) 033 [hep-th/0410123] [INSPIRE].

[46] C.R. Nappi and E. Witten, A WZW model based on a nonsemisimple group, Phys. Rev. Lett. 71 (1993) 3751 [hep-th/9310112] [INSPIRE]. 
[47] A. Hashimoto and S. Sethi, Holography and string dynamics in time dependent backgrounds, Phys. Rev. Lett. 89 (2002) 261601 [hep-th/0208126] [INSPIRE].

[48] M. Spradlin, T. Takayanagi and A. Volovich, String theory in beta deformed spacetimes, JHEP 11 (2005) 039 [hep-th/0509036] [INSPIRE].

[49] J. Lukierski, H. Ruegg, A. Nowicki and V.N. Tolstoi, Q deformation of Poincaré algebra, Phys. Lett. B 264 (1991) 331 [INSPIRE].

[50] J. Lukierski and A. Nowicki, Quantum deformations of $D=4$ Poincaré and Weyl algebra from $Q$ deformed $D=4$ conformal algebra, Phys. Lett. B 279 (1992) 299 [INSPIRE].

[51] M.A. Semenov-Tian-Shansky, What is a classical r-matrix?, Funct. Anal. Appl. 17 (1983) 259 [INSPIRE].

[52] M.A. Semenov-Tian-Shansky, Integrable Systems and Factorization Problems, nlin/0209057.

[53] V.N. Tolstoy, Twisted Quantum Deformations of Lorentz and Poincaré algebras, Bulg. J. Phys. 35 (2008) 441 [arXiv:0712.3962].

[54] S. Zakrzewski, Poisson structures on the Lorentz group, Lett. Math. Phys. 32 (1994) 11.

[55] S. Zakrzewski, Poisson structures on the Poincaré group, Comm. Math. Phys. 185 (1997) 285 [q-alg/9602001].

[56] H. Liu, G.W. Moore and N. Seiberg, Strings in time dependent orbifolds, JHEP 10 (2002) 031 [hep-th/0206182] [INSPIRE].

[57] A. Borowiec, J. Lukierski, M.n. Mozrzymas and V.N. Tolstoy, $N=1 / 2$ Deformations of Chiral Superspaces from New Twisted Poincaré and Euclidean Superalgebras, JHEP 06 (2012) 154 [arXiv:1112.1936] [INSPIRE].

[58] M. Hatsuda and S. Mizoguchi, Nonlocal charges of T-dual strings, JHEP 07 (2006) 029 [hep-th/0603097] [INSPIRE].

[59] S. Kachru, X. Liu and M. Mulligan, Gravity duals of Lifshitz-like fixed points, Phys. Rev. D 78 (2008) 106005 [arXiv:0808.1725] [InSPIRE].

[60] H. Kyono and K. Yoshida, Yang-Baxter invariance of the Nappi-Witten model, arXiv:1511.00404 [INSPIRE].

[61] S. Schäfer-Nameki, M. Yamazaki and K. Yoshida, Coset Construction for Duals of Non-relativistic CFTs, JHEP 05 (2009) 038 [arXiv:0903.4245] [INSPIRE]. 